\title{
Metanálise da relação entre espessura de toicinho e variáveis nutricionais de porcas gestantes e lactantes
}

\author{
Meta-analysis of relation among backfat thickness and nutritional variables of gestating and \\ lactating sows
}

\author{
Carlos Augusto Rigon Rossi ${ }^{I}$ Paulo Alberto LovattoII Volnei Antônio Weschenfelder ${ }^{\text {III }}$ \\ Cheila Roberta Lehnen ${ }^{\mathrm{III}}$ Bruno Neutzling Fraga ${ }^{\mathrm{IV}}$ Ines Andretta $^{\mathrm{IV}}$ Marcos Speroni Ceron $^{\mathrm{IV}}$
}

RESUMO

Uma metanálise foi realizada para avaliar a relação entre a espessura de toicinho e as variáveis nutricionais de porcas gestantes e lactantes. A base de dados contemplou 14 artigos publicados de 2000 a 2006 em revistas indexadas. A metanálise foi realizada através de análises gráfica, de correlação e de variância. A correlação entre espessura de toicinho na gestação (ETg) com consumo de alimento na gestação (CAl G) foi de -0,09 $(P=0,04)$, com CAl na lactação (CAl L) baseado na tabela NRC (1998) foi de -0,09 $(P=0,06)$, com CAl na lactação baseado na tabela Rostagno (2000) foi de -0,08 $(P=0,06)$ e com consumo de proteína bruta na lactação (CAl L PB) foi de -0,08 (P=0,06). A correlação entre espessura de toicinho na lactação (ETl) com CAl na gestação foi de 0,29 (P<0,01), com CAl na gestação NRC (1998) foi de 0,20 ( $P<0,01)$, com CAl na gestação Rostagno (2000) foi de $0,20$ ( $P<0,01)$, com $C A l$ na lactação foi de $-0,21 \quad(P<0,01)$, com consumo na lactação NRC (1998) foi de -0,24 $(P<0,01)$ e com consumo na lactação Rostagno (2000) foi de 0,24 $(P<0,01)$. O CAl de PB na gestação foi de 0,20 $(P<0,01)$ e o $C A l$ de $P B$ na lactação foi de $-0,26(P<0,01)$. A variação da espessura de toicinho durante a lactação é influenciada pelos níveis de proteína e lisina da dieta e pela gordura e proteína no leite. A variação da espessura de toicinho na gestação e na lactação foi semelhante para as tabelas do NRC (1998) e de Rostagno (2000). Há relação significativa entre espessura de toicinho e variáveis nutricionais em porcas gestantes e lactantes.

Palavras-chave: consumo alimentar, lisina, proteína bruta, modelos mistos.

\section{ABSTRACT}

A meta-analysis was undertaken to evaluate the relationship between backfat thickness and nutritional variables of sows during gestation and lactation. The database was developed from 14 studies published between 2000 and 2006. The meta-analysis was carried out trough by graphical analysis, correlation and variance. The correlation between backfat thickness at gestation (BTg) and feed intake (FI) at this phase was -0.09 $(P=0.04)$. The correlation between backfat thickness at gestation (BTg) and lactation FI NRC (1998) was -0.09 $(P=0.06) ; B T g$ and FI at lactation Rostagno (2000) presented a correlation of-0.08 $(P=0.06)$. In the same way the correlation between $B T$ and crude protein $(C P)$ intake at lactation was $0.08(P=0.06)$. The correlation between backfat thickness at lactation (BTl) and FI at gestation was $0.29(P<0.01)$. BTl and FI at gestation NRC (1998) presented a correlation of 0.20 $(P<0.01)$. For BTl and gestation feed intake Rostagno (2000) a correlation of $0.20(P<0.01)$ was reported. The correlation between BTl and lactation FI was $-0.21(P<0.01)$, the correlations between BTl and lactation FI NRC (1998) were $0.24(P<0.01)$ and lactation FI Rostagno (2000) $0.24(P<0.01)$. Correlations of $0.20(P<0.01)$ and $-0.26(P<0.01)$ were observed between $\mathrm{BTl}$ and $\mathrm{CP}$ intake at gestation and CP intake at lactation respectively. The backfat thickness variation at lactation is influenced by protein and lysine levels in the diet and fat and protein of the milk. Gestation and lactation backfat thickness variation was similar to NRC (1998) and Rostagno (2000). There is a significant relationship between backfat thickness and nutritional variables in pregnant and farrowing sows.

Key words: crude protein, feed intake, lysine, mixed models.

\section{INTRODUÇÃO}

O sucesso da suinocultura moderna está relacionado, em grande parte, ao desempenho reprodutivo das porcas (HASHIMOTO et al., 2004). A

IPrograma de Pós-graduação em Zootecnia (PPGZ), Departamento de Zootecnia (DZ), Universidade Federal de Santa Maria (UFSM), 97105-900, Santa Maria, RS, Brasil. E-mail: carlos.rossi@hotmail.com. Autor para correspondência.

IIDZ, UFSM, Santa Maria, RS, Brasil.

IIIPPGZ, UFSM, Santa Maria, RS, Brasil.

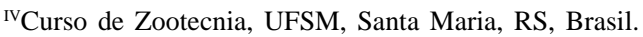


nutrição e a alimentação são os aspectos que mais afetam o desempenho reprodutivo e que mais contribuem no custo de produção, representando 65 a 85\% do custo do total de produção (YOUNG \& AHERNE, 2005a). As exigências nutricionais das porcas variam com a idade, o peso metabólico e a fase reprodutiva. No caso específico da gestação, as exigências são inferiores às da lactação e dependem da ordem de parto, do estado nutricional, do período da gestação, da estação do ano e da linhagem da fêmea (FERREIRA et al., 2006).

Na prática, as exigências nutricionais das porcas em gestação e lactação são determinadas por um escore que, fundamentado na descrição visual, quantifica e qualifica a condição corporal em índice de 1 a 5(YOUNG \& AHERNE, 2005a). A análise através do escore corporal permite classificar as porcas em grupos homogêneos, adequando-os às dietas (DOURMAD et al., 2001). No entanto, a classificação do escore apenas pela avaliação visual é um critério subjetivo, o que pode gerar situações de sub ou super-alimentação (YOUNG et al., 2004). Mas a avaliação da condição corporal de porcas gestantes e lactantes pode também ser baseada em variáveis objetivas como a espessura de toicinho (ET), a ordem de parto e o peso vivo. Essas variáveis podem ser utilizadas para adequar as exigências nutricionais e reduzir o custo de alimentação (YOUNG \&AHERNE, 2005a).

Nas condições tecnológicas atuais, o uso somente de variáveis subjetivas para a definição de programas alimentares e nutricionais de porcas pode não ser suficiente. Mas as informações disponíveis sobre o ajuste nutricional através de variáveis objetivas são incipientes e pouco conclusivas quando analisadas de forma isolada. Como as pesquisas em ciência animal dependem de métodos estatísticos, as relações quantitativas podem ser mais bem exploradas através da metanálise. Este método permite combinar os resultados de estudos realizados de forma independente (extraídos de trabalhos publicados) e sintetizar suas conclusões ou mesmo extrair uma nova conclusão (LUIZ, 2002). Assim, este trabalho tem o objetivo de avaliar de forma sistêmica, através da metanálise, a relação entre a espessura de toicinho e as variáveis nutricionais de porcas gestantes e lactantes.

\section{MATERIAL E MÉTODOS}

O trabalho foi realizado no Setor de Suinocultura do Departamento de Zootecnia da Universidade Federal de Santa Maria e no Grupo de Modelagem Animal (Grupos de Pesquisa CNPq). Foram utilizados 14 artigos publicados de 2000 a 2006, em revistas indexadas internacionais (PRUNIER et al., 2001; SINCLAIR et al., 2001; COOPER et al., 2001; CLOWES et al., 2003a; MAES et al., 2004; RAMANAU et al., 2004; VAN DER PEET-SCHWERING et al., 2004; YOUNG et al., 2004; RENSIS et al., 2005; YOUNG et al., 2005b; LANDBLOM et al., 2006) e nacionais (GUEDES \& NOGUEIRA, 2000; HASHIMOTO et al., 2004; LIMA et al., 2006). As tabelas do NRC (1998) e de ROSTAGNO et al. (2000) foram avaliadas juntamente com os resultados da metanálise. As informações relevantes do material e métodos e resultados de cada artigo foram inseridas em uma base de dados elaborada em planilha Excel. As metodologias utilizadas para definição das variáveis dependentes e independentes e para a codagem dos dados seguiram proposições descritas na literatura (LOVATTO, 2003; SAUVANT et al., 2005). Resumidamente, a codagem dos dados seguiu critérios qualitativos de agrupamento. De forma objetiva, a codagem é um recurso utilizado em metanálise para associar grupos homogêneos em determinados critérios, o que permite incluí-los nos modelos analíticos como fonte de variação. As principais codagens foram a espessura de toicinho (ETco), os tratamentos (TRATco), o peso vivo (PVco) e a ordem de parto (OPco). A base de dados utilizada na metanálise totalizou 560 tratamentos, com média de 24 animais cada (variação de 7 a 324), 12.635 porcas gestantes e lactantes, espessura de toicinho média de $17,4 \mathrm{~mm}$ (variação de 4,5 a 28,1), avaliada no final da gestação, período de lactação de 21 dias, peso médio de $235 \mathrm{~kg}$ (variação de 149 a 334) e a ordem de parto foi distribuída em três grupos (prímíparas, de dois a três partos e acima de três partos). A metanálise foi realizada através de três análises seqüenciais: gráfica, de correlação e de variância (SAUVANT et al., 2005). Na análise gráfica, foi estudada a coerência biológica dos dados, propriedade em que o pesquisador pode avaliar pelo seu conhecimento acumulado se as informações sistêmicas da base de dados têm coerência com as respostas biológicas esperadas. $\mathrm{Na}$ análise de correlação, foram identificadas as variáveis correlacionadas na base. Na análise de variância (ANOVA), foram incluídos no modelo os fatores que variaram em função da significância das correlações. As equações de regressão foram obtidas através da covariância contínua nos modelos de ANOVA. As análises descritivas e estatísticas foram realizadas através do programa MINITAB (MCKENZIE \& GOLDMAN, 1999).

\section{RESULTADOS E DISCUSSÃO}

Os coeficientes de correlação entre a espessura de toicinho na gestação (ETg), a variação 
na espessura de toicinho na lactação (ETl) e as variáveis nutricionais das porcas gestantes e lactantes são apresentados na tabela 1 . Os resultados de correlação são os de metanálise e os obtidos com as tabelas do NRC e de Rostagno. Houve correlação positiva $(\mathrm{P}=0,04)$ entre ETg e CAl na gestação, diferentemente dos resultados, obtidos das tabelas do NRC $(\mathrm{P}=0,64)$ e Rostagno $(\mathrm{P}=0,64)$. A correlação foi positiva $(\mathrm{P}<0,01)$ entre ETl e CAl na gestação, entre ETl e CAl na gestação com a tabela do NRC e entre ETl e CAl na gestação com a tabela de Rostagno. As exigências energéticas de porcas na gestação são distribuídas para a mantença, os produtos da concepção e o ganho de peso materno (YOUNG et al., 2005b). Dessas exigências, mais de $75 \%$ são destinados para mantença, o que implica a necessidade de estimá-la precisamente (JI et al., 2005).

É importante observar que as exigências energéticas para porcas na gestação diferem a partir do peso corporal (número de partos, genótipo, etc.) dos animais, do ambiente (temperatura, atividade), de perda de peso na lactação anterior e do ganho materno estimado (NOBLET et al., 1990). A avaliação do ganho corporal na gestação é recomendada, pois animais com ETg acima de $21 \mathrm{~mm}$ no parto apresentam baixo consumo de alimento, aumentam a deposição de gordura na glândula mamária, perdem mais peso na lactação e diminuem o desempenho reprodutivo subseqüente (YOUNG et al., 2005b). O aumento da ETg de 17,9 para $24,3 \mathrm{~mm}$ diminuiu em $1,6 \mathrm{~kg} \mathrm{dia}^{-1}$ o consumo voluntário de alimento na lactação para primíparas
(REVELL et al., 1998). Assim, para maximizar o desempenho e a longevidade das porcas, é fundamental ajustar o consumo alimentar às reservas corporais para evitar situações de obesidade ou emagrecimento excessivos (YOUNG \& AHERNE, 2005a).

A correlação foi positiva $(\mathrm{P}<0,01)$ entre ETl e $\mathrm{CAl}$ na gestação. Esse efeito $(\mathrm{P}<0,01)$ também foi observado entre ETl e CAl na gestação, baseado nas tabelas NRC e Rostagno, respectivamente. O consumo alimentar na gestação deve ser suficiente para o crescimento fetal, no último terço da gestação, para o ganho materno protéico e lipídico. No entanto, as alterações no peso do tecido materno e nos produtos da concepção variam durante a gestação (JI et al., 2005). A estratégia alimentar das porcas, entretanto, deve ser flexível para adequar-se às necessidades energéticas. A metanálise apresentou maior correlação entre ETl e consumo de alimento na gestação do que os resultados baseados nas tabelas NRC e Rostagno. Esse resultado reforça a importância da variável CAl na gestação, a qual pode ser usada no ajuste de programas alimentares de porcas gestantes e lactantes.

A correlação foi positiva $(\mathrm{P}=0,06)$ entre ETg e CAl na lactação NRC e entre ETg e CAl na lactação Rostagno. Houve correlação positiva $(\mathrm{P}<0,01)$ entre ETl e CAl na lactação, entre ETl e CAl na lactação NRC e entre ETl e CAl na lactação Rostagno. O consumo alimentar adequado na gestação aumenta as reservas corporais ao parto, ajuda porcas altamente prolíferas a adaptarem-se à demanda nutricional na lactação e melhora o desempenho reprodutivo subseqüente

Tabela 1 - Coeficientes de correlação entre espessura de toicinho e variáveis nutricionais de porcas gestantes e lactantes.

\begin{tabular}{|c|c|c|c|c|}
\hline \multirow{2}{*}{ Resposta } & \multicolumn{4}{|c|}{ Variáveis } \\
\hline & $\mathrm{CAl} \mathrm{G} \mathrm{g} \mathrm{d}{ }^{-1}$ & CAl G NRC, $\mathrm{g} \mathrm{d}^{-1}$ & CAl G Rostagno, $\mathrm{g} \mathrm{d}^{-1}$ & CAl G lisina, $\mathrm{g} \mathrm{d}^{-1}$ \\
\hline ETg & $-0,098$ & $-0,022$ & $-0,022$ & $-0,026$ \\
\hline $\mathrm{P}$ & 0,040 & 0,647 & 0,647 & 0,585 \\
\hline $\mathrm{ETl}$ & 0,295 & 0,201 & 0,201 & 0,211 \\
\hline \multirow[t]{2}{*}{$\mathrm{P}$} & 0,000 & 0,002 & 0,002 & 0,001 \\
\hline & CAl L PB, $\mathrm{g} \mathrm{d}^{-1}$ & CAl L, $\mathrm{g} \mathrm{d}^{-1}$ & CAl L NRC, $\mathrm{g} \mathrm{d}^{-1}$ & CAl L Rostagno, $\mathrm{g} \mathrm{d}^{-1}$ \\
\hline ETg & $-0,023$ & $-0,069$ & $-0,089$ & $-0,089$ \\
\hline $\mathrm{P}$ & 0,626 & 0,145 & 0,062 & 0,062 \\
\hline ETl & 0,201 & $-0,213$ & $-0,242$ & $-0,242$ \\
\hline \multirow[t]{2}{*}{$\mathrm{P}$} & 0,002 & 0,001 & 0,000 & 0,000 \\
\hline & CAl L lisina, $\mathrm{g} \mathrm{d}^{-1}$ & CAl L PB, $\mathrm{g} \mathrm{d}^{-1}$ & Gordura leite, \% & Proteína leite, \% \\
\hline ETg & $-0,018$ & $-0,088$ & 0,098 & 0,379 \\
\hline $\mathrm{P}$ & 0,701 & 0,066 & 0,040 & 0,182 \\
\hline $\mathrm{ETl}$ & $-0,261$ & $-0,260$ & $-0,398$ & $-0,875$ \\
\hline $\mathrm{P}$ & 0,000 & 0,000 & 0,000 & 0,001 \\
\hline
\end{tabular}

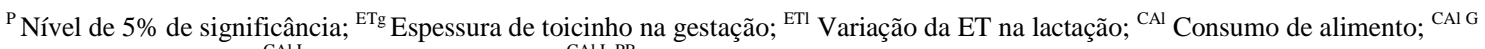
Consumo na gestação; ${ }^{\mathrm{CAl} \mathrm{L}}$ Consumo na lactação; ${ }^{\mathrm{CAl} \mathrm{L} \mathrm{PB}}$ Consumo de proteína bruta na lactação. 
(PRUNIER et al., 2001). No entanto, porcas que consomem grandes quantidades de alimento na gestação, apresentam maiores reservas de gordura corporal e maiores níveis de leptina ao parto (BARB, 1999). A leptina, originada no tecido adiposo, pode atuar no cérebro, como um dos mediadores na regulação do apetite na lactação (PRUNIER et al., 2001). Outra hipótese sobre a regulação do apetite é a hipofagia pós-parto em porcas obesas, justificada pela maior resistência à insulina e pela baixa tolerância à glicose (WELDON et al., 1994). O resultado são níveis elevados de glicose após o consumo alimentar, retardando o início da refeição seguinte. Além disso, a resistência à insulina favorece a mobilização de nutrientes do organismo, também reduzindo o consumo alimentar (PRUNIER et al., 2001). Assim, porcas com maior reserva de gordura ao parto apresentam redução no consumo voluntário de alimento na lactação, podendo aumentar o déficit nutritivo (ESTIENNE et al., 2000).

A correlação foi positiva $(\mathrm{P}<0,01)$ entre ETl e consumo de proteína bruta (PB) na gestação e entre ETl e consumo de PB na lactação. Houve correlação positiva $(\mathrm{P}=0,06)$ entre ETg e consumo de $\mathrm{PB}$ na lactação. Uma dieta com baixo nível protéico na gestação parece adequada às exigências nutricionais, mas, na lactação, não supre as necessidades energéticas (MC MILLAN et al., 2002). A perda de peso vivo materno na lactação é composta por tecido adiposo e proteína (CLOWES et al., 2003a). A mobilização das reservas corporais, na lactação, ocorre independentemente da dieta. Uma mobilização de 9 a $12 \%$ de massa protéica ao parto não representa conseqüências para o crescimento da leitegada (CLOWES et al., 2003b). Entretanto, a diminuição de mais de $12 \%$ da massa protéica causa queda na concentração de proteína no leite, no crescimento de leitegada e nas variáveis reprodutivas futuras (CLOWES et al., 2003a). A correlação significativa da PB com ETg e ETl demonstra que essa variável é importante no ajuste nutricional das porcas.

A correlação foi positiva $(\mathrm{P}<0,01)$ entre ETl e consumo de lisina na gestação e para ETl e consumo de lisina na lactação. A lisina, considerada o aminoácido limitante para suínos, é utilizada como referência para a formulação de rações (COTA et al., 2003). Ela é usada na quantidade de 45 a 48g/dia para minimizar a mobilização de massa protéica de primíparas na lactação (TOUCHETTE et al., 1998). As primíparas que consomem baixas quantidades de lisina perdem mais peso na lactação que se alimentadas com altos níveis desse aminoácido (COTA et al., 2003). A correlação significativa entre ETg e lisina sugere que os níveis desse aminoácido na dieta contribuem para o ajuste de programas nutricionais de porcas gestantes e lactantes.

A correlação foi positiva $(\mathrm{P}<0,01)$ entre ETl e a percentagem de gordura no leite e para ETl e a percentagem de proteína no leite. O conteúdo de gordura e proteína no leite é influenciado pela dieta lactacional; logo, níveis elevados de proteína na dieta resultam em maior percentagem de gordura e proteína no leite (SINCLAIR et al., 2001).

Os resultados para espessura de toicinho na gestação (ETg) obtidas por análise de covariância contínua utilizando covariáveis nutricionais são apresentados na tabela 2. A variável resposta apresentou coeficientes de correlação semelhantes nas diferentes codagens e covariáveis utilizadas na análise. O consumo na gestação apresentou o menor coeficiente de correlação $(\mathrm{R} 2=32 \%)$, quando foi utilizada a ETco (ETg=17,7-0,001 consumo na gestação,

Tabela 2 - Equações para espessura de toicinho na gestação (ETg) obtidas por análise de covariância contínua utilizando covariáveis nutricionais.

\begin{tabular}{|c|c|c|c|c|c|c|}
\hline Resposta & Fatores & Covariáveis & $\mathrm{N}$ & dpr & $\mathrm{R}^{2}, \%$ & Equação \\
\hline ETg & ETco & $\mathrm{CAl}, \mathrm{g} \mathrm{d}^{-1}$ & 441 & 3,25 & 32,44 & $\mathrm{ETg}=17,68-0,001 \mathrm{CAl}, \mathrm{g} \mathrm{d}^{-1}$ \\
\hline ETg & ETco & $\mathrm{CAl} G, \mathrm{~g} \mathrm{~d}^{-1}$ & 441 & 3,24 & 32,59 & $\mathrm{ETg}=17,18-0,0001 \mathrm{CAl} \mathrm{L}, \mathrm{g} \mathrm{d}^{-1}$ \\
\hline ETg & OPco & $\mathrm{CAl} G, \mathrm{~g} \mathrm{~d}^{-1}$ & 441 & 3,23 & 32,73 & $\mathrm{ETg}=17,62-0,001 \mathrm{CAl} \mathrm{G}, \mathrm{g} \mathrm{d}^{-1}$ \\
\hline ETg & ETco & CPB G, $g^{-1}$ & 441 & 3,23 & 32,82 & $\mathrm{ETg}=17,18-0,001 \mathrm{CPB} G, \mathrm{~g} \mathrm{~d}^{-1}$ \\
\hline ETg & OPco & $\mathrm{CAl} \mathrm{G}, \mathrm{g} \mathrm{d}^{-1}$ & 441 & 3,22 & 32,87 & $\mathrm{ETg}=17,14-0,0001 \mathrm{CAl} \mathrm{L}, \mathrm{g} \mathrm{d}^{-1}$ \\
\hline ETg & OPсо & CPB L, $\mathrm{g} \mathrm{d}^{-1}$ & 441 & 3,22 & 33,10 & $\mathrm{ETg}=17,15-0,001 \mathrm{CPB} \mathrm{L}, \mathrm{g} \mathrm{d}^{-1}$ \\
\hline ETg & ETco & CAl L e CL L, g d ${ }^{-1}$ & 441 & 3,23 & 33,15 & $\mathrm{ETg}=17,22-0,0006 \mathrm{CAl} \mathrm{L}+0,06 \mathrm{CL} \mathrm{L}, \mathrm{g} \mathrm{d}^{-1}$ \\
\hline ETg & OPco & CAl L e CL L, g d $^{-1}$ & 441 & 3,22 & 33,44 & $\mathrm{ETg}=17,18-0,0006 \mathrm{CAl} \mathrm{L}+0,06 \mathrm{CL} \mathrm{L}, \mathrm{g} \mathrm{d}^{-1}$ \\
\hline ETg & ETco & CAl G e CAl L, $g^{-1}$ & 441 & 3,22 & 33,57 & $\mathrm{ETg}=17,9-0,001 \mathrm{CAl} \mathrm{G}-0,0001 \mathrm{CAl} \mathrm{L}, \mathrm{g} \mathrm{d}^{-1}$ \\
\hline ETg & OPco & CAl G e CAl L, $\mathrm{g} \mathrm{d}^{-1}$ & 441 & 3,21 & 33,86 & $\mathrm{ETg}=17,84-0,001 \mathrm{CAl} \mathrm{G}-0,0001 \mathrm{CAl} \mathrm{L}, \mathrm{g} \mathrm{d}^{-1}$ \\
\hline
\end{tabular}

dpr Desvio padrão residual; ${ }^{\text {R2 }}$ Coeficiente de regressão; ${ }^{\text {ETg }}$ Espessura de toicinho na gestação; ${ }^{\text {ETco }}$ Codagem da ET; ${ }^{\text {CAl }}$ Consumo de alimento;

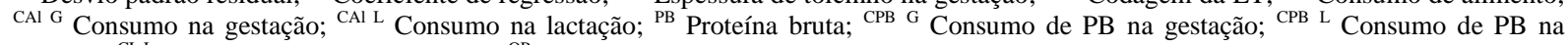
lactação; ${ }^{\mathrm{CL} L}$ Consumo de lisina na lactação; ${ }^{\mathrm{OPco}}$ Codagem da ordem de parto. 
$\left.\mathrm{g} \mathrm{d}^{-1}\right)$. Embora a média da ETg $(17,7 \mathrm{~mm})$ aproxime-se da ET ideal ao parto (18-19mm) (YOUNG \& AHERNE, 2005a), a equação apresenta uma diminuição na ETg em $0,001 \mathrm{~mm}$ para cada grama de alimento consumido. Esse resultado pode ser explicado pelo catabolismo das reservas corporais próximo ao parto, pois a ETg, em todos os trabalhos utilizados na metanálise, foi avaliada no final da gestação. Dessa forma, nas duas últimas semanas de gestação, o nível alimentar deve ser aumentado em $1 \mathrm{~kg} \mathrm{~d}^{-1}$ para evitar o balanço energético negativo e estimular as enzimas hepáticas e intestinais em função da lactogênese/produção de leite (YOUNG \& AHERNE, 2005a).

O consumo na gestação e o consumo na lactação apresentaram o maior coeficiente de correlação (R2=34\%), quando foi utilizada a OPco no modelo. A relação entre consumo na gestação e consumo na lactação é importante, pois porcas obesas ao parto apresentam custos desnecessários quanto ao ganho de peso, ETg, deposição de gordura subcutânea na glândula mamária e subseqüente baixa produção de leite (YOUNG \& AHERNE, 2005a). A maior ETg ainda diminui o consumo alimentar na lactação, reduzindo o desempenho da leitegada (YOUNG et al., 2004).

As equações para espessura de toicinho na lactação obtidas por análise de covariância contínua utilizando covariáveis nutricionais são apresentadas na tabela 3. O coeficiente de correlação entre ETl e consumo de PB na lactação e os resultados obtidos com as tabelas NRC e Rostagno foram semelhantes R2 $=55 \%$. A mobilização das reservas corporais na lactação ocorre independentemente de dieta restrita e pode comprometer a lactação e o desempenho reprodutivo subseqüente (KING et al., 1993). O declínio na produção de leite é observado em porcas que perderam grandes quantidades de sua massa protéica de origem muscular ou da qualidade dos aminoácidos liberados das reservas corporais (CLOWES et al., 2003b). Porcas com maiores reservas protéicas ao parto podem adaptar-se melhor com a dieta na lactação (CLOWES et al., 2003a). Em geral, as porcas possuem reservas corporais de proteína que variam de 24 a $30 \mathrm{~kg}$ para assegurar uma boa produção de leite (CLOWES et al., 2003b). Pela equação obtida na metanálise, para cada grama de PB consumida na lactação, há uma redução de $0,001 \mathrm{~mm}$ de espessura de toicinho. Dessa forma, as porcas necessitam de quantidades suficientes de reservas protéicas ao parto para satisfazer a demanda da produção de leite.

O consumo de lisina na lactação apresentou coeficiente de correlação de R2=66\%, quando a OPco foi utilizada na análise (ETl=2,82 - 0,028 x consumo de lisina na lactação, $\mathrm{g} \mathrm{d}^{-1}$ ). A ETl não é influenciada pelos níveis de lisina da dieta (TOUCHETTE et al., 1998). Por outro lado, há uma variação significativa da ETl, tendo um comportamento de redução linear à medida que se eleva o nível de lisina na ração de 0,6 para 1,6\% (YANG et al., 2000). Os resultados da metanálise demonstram uma diminuição da ETl em 0,028mm para cada grama de consumo de lisina na lactação.

Esta metanálise é continuação do trabalho anterior (ROSSI et al., 2008), que estudou as relações entre a espessura de toicinho e as variáveis corporais e reprodutivas de porcas gestantes e lactantes. Foram apresentados resultados da metaanálise e simulados com as tabelas do NRC e de Rostagno. A correlação entre ETg e CAl na gestação foi significativa para os resultados da metanálise, enquanto correlação entre ETg e CAl na lactação foi significativa para os resultados das tabelas NRC e Rostagno. As demais

Tabela 3 - Equações para espessura de toicinho na lactação (ETl) obtidas por análise de covariância contínua utilizando covariáveis nutricionais.

\begin{tabular}{|c|c|c|c|c|c|c|}
\hline Resposta & Fatores & Covariáveis & $\mathrm{N}$ & $\mathrm{dpr}$ & $\mathrm{R}^{2}, \%$ & Equação \\
\hline ETl & ETlco & CPB L Rostagno, $\mathrm{g} \mathrm{d}^{-1}$ & 239 & 1,22 & 55,09 & $\mathrm{ETl}=2,42-0,001$ cons L PB Rostagno, $\mathrm{g} \mathrm{d}^{-1}$ \\
\hline $\mathrm{ETl}$ & ETlco & CPB L NRC, $\mathrm{g} \mathrm{d}^{-1}$ & 239 & 1,22 & 55,09 & $\mathrm{ETl}=2,41-0,001 \mathrm{CPB}$ L NRC, $\mathrm{g} \mathrm{d}^{-1}$ \\
\hline ETl & ETlco & $\mathrm{CAl} \mathrm{L}, \mathrm{g} \mathrm{d}^{-1}$ & 239 & 1,22 & 55,3 & $\mathrm{ETl}=2,5-0,0002 \mathrm{CAl} \mathrm{L}, \mathrm{g} \mathrm{d}^{-1}$ \\
\hline ETl & ETlco & CPB L, $\mathrm{g} \mathrm{d}^{-1}$ & 239 & 1,22 & 55,49 & $\mathrm{ETl}=2,47-0, \mathrm{CPB} \mathrm{L}, \mathrm{g} \mathrm{d}^{-1}$ \\
\hline ETl & ETlco & CL L e CPB L, $\mathrm{g} \mathrm{d}^{-1}$ & 239 & 1,21 & 56,38 & $\mathrm{ETl}=2,45+0,08 \mathrm{CL} \mathrm{L}-0,005$ CPB L, $\mathrm{g} \mathrm{d}^{-1}$ \\
\hline ETl & ETlco & CAl L e CL L, g d $^{-1}$ & 239 & 1,21 & 56,41 & $\mathrm{ETl}=2,77-0,001 \mathrm{CAl} \mathrm{L}+0,13 \mathrm{CL} \mathrm{L}, \mathrm{g} \mathrm{d}^{-1}$ \\
\hline ETl & ETco & CL L, $\mathrm{g} \mathrm{d}^{-1}$ & 239 & 1,23 & 64,42 & $\mathrm{ETl}=2,66-0,02 \mathrm{CL} \mathrm{L}, \mathrm{g} \mathrm{d}^{-1}$ \\
\hline ETl & ETco & CAl L, $\mathrm{g} \mathrm{d}^{-1}$ & 239 & 1,23 & 64,44 & $\mathrm{ETl}=2,77-0,0002 \mathrm{CAl} \mathrm{L}, \mathrm{g} / \mathrm{d}$ \\
\hline $\mathrm{ETl}$ & OPco & $\mathrm{CL} \mathrm{L}, \mathrm{g} \mathrm{d}^{-1}$ & 239 & 1,20 & 66,16 & $\mathrm{ETl}=2,82-0,028 \mathrm{CL} \mathrm{L}, \mathrm{g} \mathrm{d}^{-1}$ \\
\hline $\mathrm{ETl}$ & OPco & $\mathrm{CAl} \mathrm{L}, \mathrm{g} \mathrm{d}^{-1}$ & 239 & 1,20 & 66,18 & $\mathrm{ETl}=2,93-0,0002 \mathrm{CAl} \mathrm{L}, \mathrm{g} \mathrm{d}^{-1}$ \\
\hline
\end{tabular}

dpr Desvio padrão residual; ${ }^{R 2}$ Coeficiente de regressão; ${ }^{\text {ETl }}$ Variação da ET na lactação; ${ }^{\text {ETco }}$ Codagem da ET; ETlco Codagem da ETl; ${ }^{\text {CAl G }}$ Consumo na gestação; ${ }^{\mathrm{CAl} L}$ Consumo na lactação; ${ }^{\text {OPco }}$ Codagem da ordem de parto; ${ }^{\text {CAl }}$ Consumo de alimento; ${ }^{C L} \mathrm{~L}$ Consumo de lisina na lactação. 
correlações envolvendo ETl e CAl na lactação foram semelhantes. As correlações entre ETl e consumo de PB na gestação e lactação foram significativas em relação à ETg e ao consumo de PB na lactação. Já a correlação entre a ETl e o consumo de lisina na gestação e lactação foram semelhantes. A esse conjunto de dados podem ser traçados inúmeros pontos de vista, com distintos objetivos e hipóteses, que resultarão em conclusões originais ou mais sólidas em relação às conclusões anteriores.

\section{CONCLUSÕES}

A metanálise permite abordar de forma sistemática a análise conjunta de trabalhos realizados de forma independente. A confiabilidade da metanálise reside tanto na aplicação de técnicas estatísticas apropriadas para sintetizar a informação de estudos distintos, quanto à quantidade e, sobretudo, qualidade dos mesmos.

Nesta metanálise, ficou evidenciada que a variação da espessura de toicinho na lactação ocorre devido ao consumo de alimento na gestação, ao consumo de alimento na lactação, aos níveis de proteína e lisina da dieta e ao conteúdo de gordura e proteína no leite.

A variação da espessura de toicinho na gestação foi semelhante para as tabelas do NRC e de Rostagno. No entanto, houve maior correlação entre a variação da espessura de toicinho na lactação e o consumo alimentar na gestação para os resultados da metanálise. Já o consumo de alimento na lactação apresentou maior correlação da variação da espessura de toicinho na lactação para os resultados das tabelas NRC e Rostagno.

\section{AGRADECIMENTOS}

À Coordenação de Aperfeiçoamento de Pessoal de Nível Superior (CAPES), pela concessão de bolsa aos mestrandos do Programa de Pós-graduação em Zootecnia da Universidade Federal de Santa Maria (UFSM) Cheila Roberta Lehnen e Volnei Antônio Weschenfelder. Ao Grupo de Modelagem Animal (Grupos de Pesquisa CNPq), pela infra-estrutura para realização do trabalho.

\section{REFERÊNCIAS}

BARB, C.R. The brain-pituitary-adipocyte axis: role of leptin in modulating neuroendocrine function. Journal of Animal Science, v.77, p.1249-1257, 1999.

CLOWES, E.J. et al. Selective protein loss in lactating sows is associated with reduced litter growth and ovarian function. Journal of Animal Science, v.81, p.753-764, 2003a.

CLOWES, E.J. et al. Parturition body size and body protein loss during lactation influence performance during lactation and ovarian function at weaning in first-parity sows. Journal of Animal Science, v.81, p.1517-1528, 2003b.
COOPER, D.R. et al. Effect of nutrient intake in lactation on sow performance: determining the threonine requirement of the high-producing lactating sow. Journal of Animal Science, v.79, p.2378-2387, 2001.

COTA, T.S. et al. Níveis de lisina em ração de lactação para fêmeas suínas primíparas. Revista Brasileira de Zootecnia, v.32, p.111-122, 2003.

DOURMAD, J.Y. et al. Mesurer l'épaisseur de lard dorsal des truies pour définir leurs programmes alimentaires. INRA Productions Animales, v.14, p.41-50, 2001.

ESTIENNE, M.J. et al. Concentrations of leptin in serum and milk collected from lactating sows differing in body condition. Domestic Animal Endocrinology, v.19, p.275-280, 2000.

FERREIRA, A.S. et al. Níveis de proteína bruta na ração para porcas pluríparas em gestação. Revista Brasileira de Zootecnia, v.35, p.761-767, 2006.

GUEDES, R.M.C.; NOGUEIRA, R.H.G. Relationship among body condition at parturition, decrease of backfat thickness and weight during the lactation and the interval from weaning to oestrus of sows. Brazilian Journal of Veterinary Research and Animal Science, v.37, p.43-46, 2000.

HASHIMOTO, F.A. et al. Níveis de proteína bruta na ração de gestação para porcas de segundo e terceiro ciclos reprodutivos. Revista Brasileira de Zootecnia, v.33, p.365-374, 2004.

JI, F. et al. Changes in weight and composition in various tissues of pregnant gilts and their nutritional implications. Journal of Animal Science, v.83, p.366-375, 2005.

KING, R. H. et al. The response of first-litter sows to dietary protein level during lactation. Journal of Animal Science, v.71, p.2457-2463, 1993.

LANDBLOM, D.G. et al. An evaluation of the replacement value of field peas for soybean meal in sow lactation diets. 2006, Capturado em 2006. Online. Disponível na internet: http://www.ag.ndsu.nodak.edu/dickinso/research/2000/ swine00b.htm.

LIMA, K.R.S. et al. Desempenho de porcas alimentadas durante a gestação, do primeiro ao terceiro parto, com rações com diferentes níveis de proteína bruta. Revista Brasileira de Zootecnia, v.35, p.1999-2006, 2006.

LOVATTO, P.A. Princípios de modelagem e sua aplicação no estudo de cadeias de produção agrícola. In: REUNIÃO ANUAL DA SOCIEDADE BRASILEIRA DE ZOOTECNIA, 2003, Santa Maria, RS. Anais. Santa Maria: SBZ, 2003. p.1-22.

LUIZ, A.J.B. Meta-análise: definição, aplicações e sinergia com dados espaciais. Cadernos de Ciência \& Tecnologia, v.19, p.407-428, 2002.

MAES, D.G.D. et al. Back fat measurements in sows from three commercial pig herds: relationship with reproductive efficiency and correlation with visual body condition scores. Livestock Production Science, v.91, p.57-67, 2004.

MC MILLAN, D.J. et al . Low protein diets: effect on sow performance. Advances in Pork Production, v.13, p.1, 2002. 
MCKENZIE, J.; GOLDMAN, R.N. The student edition of minitab for windows manual. V.12. Softcover ed. Belmont: Addison-Wesley Longman, Incorporated, 1999. 592p.

NOBLET, J. et al. Energy utilization in pregnant and lactating sows: modeling of energy requirements. Journal of Animal Science, v.68, p.562-572, 1990.

NRC. Nutrient requirements of swine. 1998. V.10, 189p.

PRUNIER, A. et al. Influence of feed intake during pregnancy and lactation on fat body reserve mobilisation, plasma leptin and reproductive function of primiparous lactating sows. Reproduction Nutritional Development v.41, p.333, 2001.

RAMANAU, A. et al. Supplementation of sows with L-Carnitine during pregnancy and lactation improves growth of the piglets during the suckling period through increased milk production. Americam Society for Nutritional Sciences, v.134, p.8692, 2004.

RENSIS, F.D. et al. Relationships between backfat depth and plasma leptin during lactation and sow reproductive performance after weaning. Animal Reproduction Science, v.90 p.95100, 2005.

REVELL, D.K. et al. Body composition at farrowing and nutrition during lactation affect the performance of primiparous sows: I. Voluntary feed intake, weight loss, and plasma metabolites. Journal of Animal Science, v.76, p.1729-1737, 1998.

ROSSI, C.A.R. et al. Metanálise da relação entre espessura de toicinho e variáveis corporais e reprodutivas de porcas gestantes e lactantes. Ciência Rural, v.38, n.1, p.206-212, 2008.

ROSTAGNO, H.S. et al. Tabelas brasileiras para aves e suínos-composição de alimentos e exigências nutricionais. Viçosa: UFV, Departamento de Zootecnia, 2000. 141 p.
SAUVANT, D. et al. Les méta-analyses des données expérimentales: applications en nutrition animale. INRA Productions Animales, v.18, p.23-33, 2005.

SINCLAIR, A.G. et al. The influence of gestation feeding strategy on body composition of gilts at farrowing and response to dietary protein in a modified lactation. Journal of Animal Science, v.76, p.2397-2405, 2001.

TOUCHETTE, K.J. et al. The lysine requirement of lactating primiparous sows. Journal of Animal Science, v.76, p.10911097, 1998.

VAN DER PEET-SCHWERING, C.M.C. et al. Effects of additional starch or fat in late-gestating high nonstarch polysaccharide diets on litter performance and glucose tolerance in sows. Journal of Animal Science, v.82, p.2964-2971, 2004.

WELDON, W.C. et al. Postpartum hypophagia in primiparous sows: I. Effects of gestation feeding level on feed intake, feeding behavior, and plasma metabolite concentrations during lactation. Journal of Animal Science, v.72, p.387-394, 1994.

YANG, H. et al. Effects of dietary lysine intake during lactation on blood metabolites, hormones, and reproductive performance in primiparous sows. Journal of Animal Science, v.78, p.1001-1009, 2000

YOUNG, M. G. et al. Comparison of three methods of feeding sows in gestation and the subsequent effects on lactation performance. Journal of Animal Science, v.82, p.30583070, 2004

YOUNG, M.; AHERNE, F. Monitoring and maintaining sow condition. Advances in Pork Production, v.16, p.299-313, 2005a.

YOUNG, M.G. et al. Effect of sow parity and weight at service on target maternal weight and energy for gain in gestation. Journal of Animal Science, v.83, p.255-261, 2005 b. 